\title{
GAGUEIRA NA WEB: QUALIDADE DA INFORMAÇÃO
}

\author{
Stuttering in the web: quality of information
}

\author{
Priscilla Carla Menezes Silveira ${ }^{(1)}$, Antônia Eliana dos Santos Costa ${ }^{(2)}$, Cleone Cassemiro de Lima ${ }^{(3)}$
}

\section{RESUMO}

Objetivo: avaliar as informações sobre gagueira em sites brasileiros na rede mundial de computadores visando, especificamente, investigar a qualidade das informações nos sites sobre gagueira e averiguar a natureza e conteúdo das informações dos sites. Método: tratou-se de um estudo descritivo. Os sites foram selecionados por meio de uma busca no Google, por página somente no Brasil, usando como termo de busca "gagueira". Os 5 sites elegíveis foram averiguados quanto a sua natureza e conteúdo das informações e avaliados quanto à qualidade dessas informações, utilizando-se dos critérios da HONcode, por 2 pesquisadores, onde foi utilizado o índice de concordância de Kappa. Resultados: verificou-se principalmente que nenhum dos sites avaliados segue as exigências de todos os critérios. Conclusão: não há qualidade em suas informações de acordo com os critérios propostos pela HONcode (2009).

DESCRITORES: Gagueira; Internet; Qualidade; Informação

\section{INTRODUÇÃO}

A humanidade vive em crescente busca por informação em saúde, inclusive sobre a gagueira, patologia esta que gera muitas dúvidas, levando a população a uma frequente busca por respostas, principalmente por se tratar de uma desordem da comunicação cujo conhecimento científico é pautado em vários conceitos e teorias divergentes.

A internet permite um acesso livre a um grande número de conhecimentos que anteriormente eram inacessíveis, além de transcender barreiras geográficas e culturais em diversas línguas, criando oportunidades de comunicação e tomadas de decisões em saúde. No entanto, estas informações podem acarretar prejuízos, tanto para a população quanto para os profissionais de saúde, pois elas advêm tanto do conhecimento do senso comum (o que pode gerar desinformação) quanto do conhecimento científico.

(1) Fonoaudióloga pela Universidade Católica de Pernambuco; Especialista em linguagem pelo Conselho Federal de Fonoaudiologia; Mestre em lingüística pela Universidade Federal de Pernambuco; Doutora em educação pela Universidade Federal do Rio Grande do Norte.

(2) Fonoaudióloga pela Universidade Potiguar; Especializanda em linguagem pela Universidade Potiguar.

(3) Fonoaudióloga pela Universidade Potiguar; Especializanda em linguagem pela Universidade Potiguar.

Conflito de interesses: inexistente
Assim, torna-se necessário que haja uma avaliação prévia do material que os sites específicos da saúde disponibilizam na rede mundial de computadores.

A Internet nasceu em 1969, nos Estados Unidos, com a função de interligar laboratórios de pesquisa e evoluiu constantemente desde a sua criação até os dias atuais. "O dinamismo dos meios de produção e comunicação, no acesso aos estoques mundiais de informação possibilitados pela era da tecnologia, exercem grande influência nas relações sociais" ${ }^{1 .}$

Não existe um método capaz de aferir, com precisão ou estatística, sobre o número de usuários da rede. Em geral é apenas possível estimar o tamanho mínimo da internet. Para se ter uma idéia da velocidade com que cresce a internet, segundo o IBOPE, o número de sites subiu de $18 \mathrm{mil} \mathrm{em}$ 1995 para 1 milhão em 1997, 10 milhões em 2000 e, em julho de 2001 , foram registrados 30 milhões de sites ${ }^{1,2}$.

Uma pesquisa realizada pelo Instituto Brasileiro de Opinião Pública e Estatística (IBOPE) indicou que existem cerca de 64,8 milhões de brasileiros, considerando a idade de 16 anos ou mais, com acesso à internet em qualquer ambiente (residências, escolas, lan-houses, bibliotecas e telecentros). Estes dados corroboram para o aumento de pesquisas pessoais e disseminação das informações, podendo, consequentemente, acarretar 
prejuízos, caso essas informações não venham a ser de fonte fidedigna.

Diante da revolução tecnológica e a crescente necessidade de busca por informação, a internet vem tornando-se um dos meios mais utilizados pela população, seja ela do senso comum ou científico, para ter acesso a variados temas, incluindo informações sobre saúde.

São indiscutíveis os benefícios que a tecnologia vem proporcionando à população em relação ao assunto saúde, porém as informações que estão dispostas na web nem sempre são favorecidas pelo conhecimento cientifico, o que pode acarretar um prejuízo para o pesquisador.

Os benefícios potenciais do uso da internet são óbvios. Para os profissionais da saúde, a internet pode ser uma valiosa ferramenta clínica, um outro meio pelo qual se podem trocar informações entre profissionais e pacientes. É uma fonte de informações em constante crescimento. A velocidade em que avança o conhecimento científico coloca a saúde em posição diferenciada em relação a outros campos dependentes do manuseio da informação. Os avanços científicos e tecnológicos geram uma grande mudança na forma de atuação dos profissionais e consequentemente no relacionamento com o paciente ${ }^{2,3}$.

Diante da revolução da comunicação na área da saúde, a pesquisa por essas informações na internet, a participação em fóruns virtuais e de grupos de apoio, o envio de e-mails para responsáveis desses serviços, consubstanciam formas de comunicação interativa em saúde. O aumento da disposição dos participantes em discutir sobre o assunto, riscos comportamentais, medos e incertezas amplia o acesso à informação. $O$ apoio solicitado também aumenta a capacidade das informações, sendo a sua audiência crescente, com pequeno custo.

Internet também faz propaganda de produtos, divulga boatos médicos e os mais sofisticados "pseudo-científicos" artigos. Isto faz com que seja extremamente difícil para o usuário determinar qual informação é útil e confiável, como ela pode ser avaliada, criticada ou verificada, quando deve ser ignorada, rejeitada ou apagada; quando deve ser lida, impressa, salva, ou transferida ${ }^{4}$.

"O crescente uso da comunicação interativa levanta questões sobre seu risco potencial para causar prejuízo à saúde dos utilizadores"5. Faz-se necessário ter a consciência do quanto a evolução da tecnologia pode trazer aspectos benéficos ou maléficos para a sociedade.

No que concerne à área da saúde, a maioria da população brasileira utiliza a automedicação como um processo natural e cultural, o que promove uma busca de informações sobre a saúde na web, pois essa ferramenta disponibiliza um grande conteúdo de informação ao cidadão, como assuntos relacionados a patologias e a tratamentos que antes estavam disponíveis apenas na área científica ${ }^{6,7}$, caracterizando-se como um risco

Em relação à gagueira, a informação é um fator primordial, principalmente, quando se pensa em sua prevenção. No entanto, no meio científico ainda não existe um consenso sobre a etiologia e tratamento desta patologia, tornando difícil a socialização sobre seu conhecimento para a população em geral ${ }^{8}$.

Além disso, os distúrbios da fluência da fala parecem não atrair a atenção da maioria dos fonoaudiólogos. Tal comportamento relaciona-se, provavelmente, ao número de teorias divergentes existentes sobre tal patologia e ainda à dificuldade em discriminar entre o que é informação cientifica daquela do senso comum ${ }^{9}$. Muitos são os estudos que apontam o conhecimento errôneo da população sobre a gagueira.

Analisando ${ }^{10} \mathrm{o}$ conhecimento de professores do ensino fundamental de escolas públicas sobre a gagueira e a forma como lidam com esta patologia, foi realizado um estudo em quatro escolas públicas do município de Bauru-SP, com professores do ensino fundamental.

Os resultados demonstraram que muitos professores definiram a gagueira como um distúrbio da fala no qual as características mais mencionadas foram as emocionais, admitiram também que acreditam na cura da gagueira. Quanto à forma como lidam com ela, foram mencionadas a postura facilitadora, atitude instrucional e de indiferença. As autoras concluíram que faltam informações aos professores sobre a gagueira que lhes possibilitem saber o que fazer diante de uma criança com essa patologia.

Em estudo similar, uma pesquisa teve o objetivo de investigar o conhecimento e atitude dos professores de pré-escola e da $1^{\underline{a}}$ à $8^{\underline{a}}$ série do ensino fundamental, frente aos alunos com gagueira, de escolas públicas e particulares do município de Ribeirão Preto-SP. As autoras ${ }^{11}$ verificaram que a maioria dos professores afirmou ter conhecimento sobre a gagueira, no entanto concluíram que os professores não sabem como lidar com o problema.

Uma pesquisa realizada na cidade de Natal teve o objetivo de verificar o conhecimento dos psicólogos sobre a gagueira, quanto a sua definição, classificação, etiologia e tratamento em consultórios de psicologia clínica. As autoras concluíram que há o desconhecimento desses profissionais em relação à gagueira do desenvolvimento, não havendo uma 
distinção entre um sujeito com gagueira e sujeitos com outros problemas psicológicos ${ }^{12}$

Outro estudo, realizado com profissionais e estudantes de $3^{\circ}$ e $4^{\circ}$ ano de fonoaudiologia, teve o objetivo de verificar a compreensão destes em relação aos conceitos de fluência e disfluência, aos componentes influenciadores da fluência e aos tipos de disfluência. Os resultados demonstraram que os estudantes e profissionais conceituam a fluência como uma fala livre de rupturas e disfluência como sinal de alteração, porém não como fenômeno intrínseco à fala. Consideraram ainda que a taxa de elocução (ritmo e velocidade da fala) é o componente que mais afeta o grau de fluência.

Além disso, os sujeitos pesquisados citam a ansiedade como o que mais interfere no grau de fluência (fator afetivo), atribuindo uma interferência secundária a fatores linguísticos, cognitivos e genéticos; categorizaram os tipos de disfluência como normal e gaga, alinhada com a literatura ${ }^{13}$.

Diante desta problemática, o objetivo geral deste estudo foi avaliar as informações sobre gagueira em sites brasileiros na rede mundial de computadores, visando, especificamente, investigar a natureza, o conteúdo e a qualidade das informações nos sites sobre gagueira.

\section{MÉTODO}

Tratou-se de um estudo descritivo. Foi selecionado o site de busca Google, (http:// www.google. com.br), por ser o mais utilizado mundialmente, segundo dados da Nielsen NetRatings (http://en-us. nielsen.com/rankings/insights/rankings/internet), em 2009 .

A pesquisa foi realizada no período de setembro a dezembro de 2009, procurando por páginas somente no Brasil e utilizando como palavra-chave o termo "gagueira". Assim foram encontrados 61.300 resultados, no dia 23 de setembro de 2009 , às $11 \mathrm{~h} 45 \mathrm{~min}$.

Os seguintes critérios foram utilizados para compor a amostra do estudo: foram avaliados os 100 primeiros resultados da coleta, já que geralmente os primeiros resultados obtidos em sites de busca são os mais acessados pelos usuários; para tal seleção, foi utilizada a ficha de observação 1 (Figura 1). Foram excluídos os sites que tiveram problemas técnicos no período da busca, blogs, twitter, grupos de discussão, fórum, links para vídeos ou arquivos, links repetidos (mesmo site), sites de links, como também os que não abordavam especificamente o tema "gagueira" como patologia.

Portanto, foram excluídos 38 sites que apresentaram problemas técnicos em dois dias de tentativas para acessá-los (38\% do total de resultados obtidos), 40 sites que não tratavam especificamente do tema "gagueira" $(40 \%$ do total de resultados), 08 por se tratar de blogs ( $8 \%$ do total de resultados obtidos), 02 grupos de discussão ( $2 \%$ do total de resultados obtidos), 02 fóruns ( $2 \%$ do total resultado obtido), 02 links para vídeo ( $2 \%$ do total resultado obtido) e 03 links repetidos (3\% do total de resultado obtido).

\section{FICHA DE OBSERVAÇÃO 1}

SITE:

Número:

Data:

Avaliadora:

Não aborda especificamente o tema gagueira

Problemas técnicos

Observações:

Figura 1 - Ficha de Observação 1 
Desta maneira, fizeram parte deste estudo 5 sites $-5 \%$ da amostra inicial, para serem consultados na íntegra e serem avaliados mais detalhadamente. Cada site foi avaliado de acordo com os critérios de qualidade definidos pelo HONcode (Health On the Net Foundation, instituição com sede em Genebra na Suíça e que, desde 1995, certifica página na web. Disponível em http://www.hon.ch) que são eles: autoridade (avalia quem fornece a informação), complementaridade (avalia se a informação visa apenas a apoiar e não a substituir a relação profissional $x$ paciente), confidencialidade (avalia o caráter confidencial dos dados), atribuições (avalia as referências às fontes das informações), justificativas (avalia o respaldo e comprovação de qualquer informação sobre benefícios/resultados de um tratamento), transparência na propriedade (avalia a clareza e contato para informações adicionais), transparência do patrocínio (avalia a clareza dos apoios financeiros dados ao site), honestidade da publicidade e da política editorial (avalia a indicação no site de que a publicidade é sua fonte de renda, se for o caso).

Além disso, eles foram distribuídos conforme a natureza das informações nos seguintes grupos: comercial (o objetivo principal é obtenção de lucro), organizacional (pertence a uma organização), pessoal e acadêmico (o objetivo principal é o ensino, pesquisa e extensão). Vale ressaltar que um site pode apresentar mais de um dos grupos acima referidos em sua natureza. Já quanto ao conteúdo das informações, foi identificado o que dizem os sites sobre a definição, etiologia e tratamento da gagueira.

Então as seguintes variáveis, foram pesquisadas: qualidade, natureza e conteúdo das informações dos sites. Para tal, foi utilizada uma ficha de observação 2 (Figura 2), que contemplou a verificação de todas as variáveis.

\section{FICHA DE OBSERVAÇÃO 2}

SITE:

Número:

Data:

Avaliadora:

1. Natureza das informações:

$\square$ Comercial $\square$ Organizacional $\square$ Pessoal $\square$ Acadêmico

2. Qualidade das informações:
$\square$ Autoridade
Complementaridade
Confidencialidade
Atribuições
Justificativas
Transparência na propriedade
Transparência do patrocínio
Honestidade da publicidade e da política editorial
Observações:

\section{Conteúdo das informações \\ Definição da gagueira:}

\section{Etiologia da gagueira:}

\section{Tratamento da gagueira:}

\section{Figura 2 - Ficha de Observação 2}


O processo de seleção inicial dos sites e de avaliação das variáveis para a amostra do estudo foi realizado por dois pesquisadores, onde foi utilizado o índice de concordância de Kappa (ICK), a fim de garantir a legitimidade dos resultados, onde é considerado ICK > 0,80: excelente; ICK 0,60 - 0,80: bom; ICK 0,40-0,60: regular; ICK < 0,40: ruim.

Assim, os dois pesquisadores preencheram, de forma independente, a ficha de observação 1. Com base nestas, foram tomadas decisões sobre a inclusão/exclusão do site. Para a análise das variáveis, foi utilizada a ficha de observação 2 .

Frente às discordâncias entre os pesquisadores sobre a marcação em ambas fichas, as decisões foram tomadas com base em discussão e consenso. Os dados dos formulários foram armazenados no Excel 2003, com dupla digitação de modo independente, sendo realizado procedimento de validação da mesma.

Vale salientar que cada site somente foi considerado adequado quanto à qualidade das informações se todos os itens e subitens da ficha de observação 2 fossem apresentados nos sites avaliados.

Para análise estatística, foi utilizado o software Excel 2003 para a construção de tabelas e cálculo da concordância bruta do índice de concordância kappa e respectivos intervalos de confiança de 95\% (IC95\%), sem ajuste para viés e prevalência.

Os resultados finais foram apresentados por distribuição de frequência absoluta e relativa, de forma tabelar.

\section{RESULTADOS}

Os 5 sites selecionados nesse estudo foram analisados quanto à natureza, à qualidade e ao conteúdo das informações. Para garantir a confiabilidade da análise por 2 pesquisadores, foi utilizado o índice de concordância de Kappa. Como os pesquisadores concordaram em $100 \%$ na análise dos sites quanto à natureza e ao conteúdo, o referido índice se fez necessário apenas na análise da qualidade das informações, cujo Kappa obtido foi o seguinte, de acordo com cada critério avaliado: autoridade (ICK> 0,89); complementaridade (ICK> 0,63); confidencialidade (ICK> 1.0); atribuições (ICK> 0,89); justificativas (ICK>0.63); transparência na propriedade $(I C K>1.0)$; transparência do patrocínio (ICK> 1.0); honestidade da publicidade e da política editorial $(I C K>1.0)$.

A seguir, serão dispostos os resultados de forma tabelar (04 tabelas). Quanto à sua natureza, os 5 sites foram assim classificados: 01 foi de natureza organizacional, 01 site foi de natureza pessoal e 01 acadêmico, 01 classificado como organizacional e comercial ao mesmo tempo, e 01 site não foi possível identificar a sua natureza.

A Tabela 1 apresenta a análise dos sites quanto à qualidade das informações, baseada nos critérios da HONcode.

O critério de autoridade foi apresentado em 2 dos sites avaliados, o que significa que, em 3 sites, não houve uma preocupação em declarar a autoria das informações.

O critério de complementaridade foi observado em 4 dos sites avaliados o que aponta que, na maior parte deles, as informações visam a apoiar e não a substituir a relação paciente/médico. 4 sites apresentaram o critério de confidencialidade, havendo uma preocupação na preservação da privacidade de seus visitantes/pacientes.

Ainda foi possível observar que apenas 1 site apresentou o critério de atribuição, que disponibiliza referências claras às fontes consultadas, e nenhum dos sites apresentou justificativa em suas informações. Esse critério exige respaldo com comprovação adequada e equilibrada com relação às afirmações feitas sobre benefícios de um tratamento, conforme o critério de atribuição da HONcode (2009).

Já o critério de transparência da propriedade foi identificado em 4 dos sites avaliados, dispondo a informação de forma clara e endereços de contatos

Tabela 1 - Distribuição de freqüências quanto aos critérios de avaliação da qualidade de informação dos 5 sites brasileiros específicos sobre gagueira, 2009

\begin{tabular}{lc}
\hline CRITÉRIOS (HONcode) & N \\
\hline Autoridade & 2 \\
Complementariedade & 4 \\
Confidencialidade & 4 \\
Atribuições & 1 \\
Justificativa & 0 \\
Transparência na propriedade & \multicolumn{2}{|c}{} \\
Transparência do patrocínio & 2 \\
Honestidade da publicidade e da política editorial & 2 \\
\hline
\end{tabular}


para quem deseja informação adicional. Dados relativos à transparência do patrocínio, foram encontrados em 2 sites, o que significa que, em 3 sites, não houve uma preocupação em declarar o autor da informação, bem como de disponibilizar os apoios financeiros fornecidos.

Pode-se observar que nenhum dos cinco sites avaliados apresentou na íntegra todos os critérios propostos pela HONcode aos sites de saúde, havendo uma falta de qualidade das informações dispostas pelos mesmos. A este respeito é possível citar trabalhos relacionados à informação sobre saúde na internet, alertando acerca do risco/beneficio aos utilizadores dessa ferramenta ${ }^{1,2}$.

As Tabelas 2, 3 e 4 apresentam a análise dos sites específicos de gagueira, quanto ao conteúdo das informações: definição, etiologia e tratamento da gagueira, respectivamente.

Tabela 2 - Distribuição de freqüências quanto ao que os sites brasileiros específicos sobre gagueira referem quanto à definição desta patologia, 2009

\begin{tabular}{ll}
\hline & $\mathbf{N}$ \\
\hline Transtorno da fluência gerada por uma alteração neurobiológica. & 3 \\
Combinação de disfunções de fala, comunicação e comportamento. & 1 \\
Cita sintomatologia observáveis e não observáveis. & 1 \\
É o efeito de uma forma subjetiva singular sobre a produção de fala. & 5
\end{tabular}

Tabela 3 - Distribuição de freqüências quanto ao que os sites brasileiros específicos sobre gagueira referem quanto à etiologia desta patologia, 2009

\begin{tabular}{ll}
\hline & N \\
\hline Dificuldade nos núcleos da base, determinada geneticamente. & 1 \\
Apresenta as diferentes vertentes existentes na literatura. & 1 \\
Formação da imagem de mau falante na infância. & 3 \\
\hline Total & 5 \\
\hline
\end{tabular}

Tabela 4 - Distribuição de freqüências quanto ao que os sites brasileiros específicos sobre gagueira referem quanto tratamento desta patologia, 2009

\begin{tabular}{ll}
\hline & N \\
\hline Autoterapias, dispositivos eletrônicos e técnicas de controle da gagueira. & 2 \\
Informa sobre as diversas linhas de tratamentos fonoaudiológicos. & 1 \\
Não aborda esse tema. & 1 \\
Resignificação da fala e modificação da imagem como falante. & 1 \\
\hline Total & 5 \\
\hline
\end{tabular}

Conforme a Tabela 2, dos 05 sites avaliados quanto à definição da gagueira, 03 concordaram sobre a sua definição (Transtorno da fluência gerada por uma alteração neurobiológica), definição essa pautada na etiologia desta patologia e; em contrapartida, em 1 desses sites, a definição foi elaborada levando em consideração a sua sintomatologia. Sabe-se que, ao definir uma patologia para ser socializada à população, é necessário que haja clareza e, quando divergente, torna esse processo mais difícil ${ }^{3}$

De acordo com a Tabela 3, dos 05 sites avaliados, no que se refere à etiologia da gagueira, 01 refere que a gagueira se dá por uma dificuldade nos núcleos da base, determinada geneticamente, outro apresenta as diferentes vertentes existentes na literatura, e 03 sites convergem para a mesma opinião, quando afirmam que a gagueira 
se dá a partir da formação da imagem de mau falante na infância.

Mais uma vez, é notável a divergência sobre a gagueira entre os sites, reflexo da não comprovação ainda de sua etiologia na literatura. Neste sentido, vê-se como fator positivo um dos sites apresentar todas as teorias existentes sobre a etiologia da gagueira.

Dos sites avaliados com relação ao tratamento da gagueira, 02 disponibilizam informações sobre autoterapias, dispositivos eletrônicos e técnicas de controle da gagueira; 01 informa sobre as diversas linhas de tratamento fonoaudiológico, 01 não aborda o tema, e 01 refere a ressignificação da fala e modificação da imagem de mau falante, como pode ser observado na Tabela 4.

Além de divergentes, verificou-se o quanto as informações sobre o tratamento da gagueira ainda podem representar uma incógnita para quem deseja se tratar, pois nenhum site apresentou informações científicas consistentes quanto à efetividade do tratamento. No entanto, ressalta-se, mais uma vez, como fator positivo, o site que apresentou informações sobre as diversas linhas de tratamento.

Os resultados dos 5 sites quanto ao seu conteúdo podem justificar, em parte, os resultados dos estudos que apontam o desconhecimento do senso comum com relação a gagueira ${ }^{8-12}$, partindo-se da premissa de que um dos grandes veículos informadores da população atualmente seja a internet.

\section{DISCUSSÃO}

Os organizadores e indivíduos responsáveis pela criação e manutenção dos sites, que abordem temas relacionado à saúde, devem ter a preocupação em oferecer conteúdos fidedignos, atualizados, corretos e de alta qualidade, como também garantir a privacidade dos seus usuários e respeitar os códigos de ética que regulamentam o exercício do profissional.
Nesse estudo, avaliamos as informações sobre gagueira em sites brasileiros na rede mundial de computadores, visando investigar a qualidade das informações nos sites sobre gagueira, além de averiguar a natureza e conteúdo das informações dos sites que abordam especificamente o tema gagueira como patologia.

Foram encontrados poucos sites que abordam o tema gagueira, apenas 05 no Brasil eram específicos sobre o assunto, sendo elegíveis para nossa pesquisa.

Além da divergência existente no meio científico inerente à gagueira, a falta de controle e qualidade das informações sobre essa desordem da comunicação faz com que os usuários da web, sejam profissionais de saúde ou leigos, não tenham a oportunidade de se aprofundarem no tema, resultando assim em um conhecimento frequentemente errôneo sobre a patologia em questão.

\section{CONCLUSÃO}

Neste estudo, foi verificado a existência de sites sobre gagueira de natureza organizacional, pessoal, acadêmico, organizacional e comercial ao mesmo tempo e de natureza não identificada.

Verificou-se que nenhum dos sites avaliados segue os critérios propostos pela HONcode (2009), havendo um comprometimento da qualidade da informação dispostas por eles. Isso se dá também devido à falta de controle sobre a qualidade da informação sobre esta patologia.

Pôde-se observar que, quanto ao conteúdo, em relação à definição, etiologia e tratamento da gagueira, as informações disponíveis nos sites são divergentes, o que torna difícil a socialização sobre o tema.

Diante disso, sugere-se a regulamentação de um selo de qualidade da informação sobre gagueira em sites brasileiros.

\begin{abstract}
Purpose: to evaluate the information on stuttering in Brazilian sites of the world-wide net of computers, and specifically, the quality, nature and content of information. Method: a descriptive study. The sites were selected through a search in the Brazilian Google version, using "stuttering" as search term. Evaluation of quality, nature and contents as for the selected topic was performed by using the criteria of HONcode by 2 researchers. Agreement between the two researchers was determined by the Kappa index. Results: none of the evaluated sites fulfilled all the criteria. Conclusion: the selected sites failed to reach the standards set out by the HONcode (2009).
\end{abstract}

KEYWORDS: Stuttering; Internet; Quality; Information 


\section{REFERÊNCIAS}

1. Pereira IM. O discurso sobre a política de cotas raciais no jornalismo online- Revista Fronteira: Estudos mediáticos/universidade do vale do Rio dos Sinos. 2008; X(03).

2. Juzzo LMLC. Critérios para avaliação da qualidade das informações sobre saúde disponíveis online. 2004 disponível em: http://www.fuden.es/ originales_detalle.cfm?ID_ORI(...) acesso em 31/08/2009.

3. Goulart LJ, Morgado EM, Neto MC, Boni GN, Domingues B, Tokunaga MK et al. Saúde e tecnologia da informação: Convergência e Mobilidade - Congresso Brasileiro de Informática em Saúde, 10, Florianópolis, 2006. Disponível em:<http://www.sbis.org.br/cbis/arquivos/1056. pdf.>Acesso em: 11/09/09. Healt on the net (HON) foundation. disponível em <http://www.hon.ch/ HONcode/Conduct.html > Acesso em 09/11/09 10h15min.

4. Sales ALC, Toutain LB. Aspectos que norteiam a avaliação da qualidade da informação em saúde na era da sociedade digital. In: CINFORM - Encontro Nacional de Ciência da Informação 5. 2005, Salvador. Anais eletrônicos... Salvador, 2005. Disponível em: <http://dici.ibict.br/ archive/00000487/01/AnaLidiaSales.pdf>. Acesso em: 09/11/09.

5. Dias SPO. Informática jurídica: Tecnologias da informação e comunicação na saúde: Uma possibilidade ou futuro? 2005 disponível em :http://www wiki.di.uminho.pt/twiki/pub/Education/ Archive/.../SusanaDias1.doc
6. Lopes IL. Novos paradigmas para avaliação da qualidade da informação em saúde recuperada na web. Ci. Inf. 2004;33 (1): 81-90, disponível em www. scielo.br/pdf/ci/v33n1/v33n1a10.pdf.

7. Castro RCF. Impacto da internet no fluxo da comunicação científica em saúde. Rev. Saúde Pública. 2006; 40(special issue).

8. Silveira PCM - Algumas relações entre a gagueira e a leitura sob uma perspectiva da análise de discurso. Tese de mestrado (Programa de pós-graduação em letras) Universidade Federal de Pernambuco. Recife- PE, 2003.

9. Barbosa LMG, Chiari BM. Gagueira: etiologia, prevenção e tratamento. Editora pro fono São Paulo, 1998.

10. Calais LL, Jorge TM, Pinheiro-Crenitte PA. Conhecimento dos professores do ensino fundamental sobre a gagueira. Pro-fono Revista de atualização cientifica. 2002;14(I): 23-30.

11. Ferriolli BHVM, Leitão PM, Pereira FLF. O conhecimento $\mathrm{e}$ as atitudes dos professores frente à gagueira. - JBF - Jornal Brasileiro de Fonoaudiologia, 2005.

12. Neri GKO, Azevedo PKB. O conhecimento dos psicólogos sobre gagueira: a efetividade do tratamento fonoaudiológico em jogo? Monografia (curso de fonoaudiologia) universidade potiguar Natal, 2004.

13. Oliveira AMCC, Ribeiro IM, Merlo S, Chiapetta ALML. O que fonoaudiólogos e estudantes de fonoaudiologia entendem por fluência e disfluência. Rev. CEFAC.2007;9(1): 4-6.
http://dx.doi.org/10.1590/S1516-18462012005000025

RECEBIDO EM: 18/11/2010

ACEITO EM: 12/09/2011

Endereço para correspondência:

Priscilla Silveira

Rua Goianinha, no 194, apto. 07

Nova Parnamirim Parnamirim - RN

CEP: $59150-480$

E-mail: pcmsilveira@gmail.com 